\title{
Effects of pregabalin and gabapentin on postoperative pain and opioid consumption after laparoscopic cholecystectomy
}

\author{
Mohammad Eidy ${ }^{1}$, Mohammad Reza Fazel ${ }^{1}$, Hossein Abdolrahimzadeh ${ }^{2}$, \\ Ali Reza Moravveji ${ }^{3}$, Ebrahim Kochaki ${ }^{1}$, and Mahdi Mohammadzadeh ${ }^{1}$ \\ ${ }^{1}$ Trauma Research Center, ${ }^{2}$ Shahid Beheshti Hospital, ${ }^{3}$ Social Determinants of Health Research centre, Kashan \\ University of Medical Sciences, Kashan, Iran
}

\begin{abstract}
Background: Gabapentin and pregabalin are antiepileptic drugs that are also used for chronic pain treatment. This study evaluated the effects of pregabalin and gabapentin on postoperative pain in patients undergoing laparoscopic cholecystectomy.

Methods: A total of 108 candidates for elective laparoscopic cholecystectomy were randomly assigned to gabapentin $(\mathrm{n}=36)$, pregabalin $(\mathrm{n}=36)$, and placebo $(\mathrm{n}=36)$ groups. Patients received $800 \mathrm{mg}$ of gabapentin or $150 \mathrm{mg}$ of pregabalin orally one hour before surgery. Postoperative analgesia was administered by pethidine via patient-controlled analgesia. The amount of opioid consumed, number of nausea events, vomiting, and pain scores at 2, 6, 12, and 24 hours after surgery were recorded.

Results: The gabapentin and pregabalin groups had significantly lower pain intensity than the placebo group, and pain intensity in the pregabalin group decreased more compared to the gabapentin group. The mean amount of pethidine consumption in the placebo group was significantly higher than in the gabapentin and pregabalin groups.

Conclusions: A single dose of gabapentin or pregabalin decreased postoperative pain and nausea, as well as vomiting and opioid consumption after laparoscopic cholecystectomy. Moreover, the findings revealed that pregabalin was superior to gabapentin for reducing postoperative pain.
\end{abstract}

Key Words: Cholecystectomy, Gabapentin, Pain, Pregabalin.

Corresponding author: Mohammad Reza Fazel, M.D.

Trauma Research Center, Kashan University of Medical Sciences, Matini Hospital, Amirkabir Avenue, Kashan 8719674591, Iran

Tel: 98-9132760380, Fax: 98-3155342025

Email: drmfazel@yahoo.com

ORCID: https://orcid.org/0000-0001-5944-9230

Received: March 17, 2016.

Revised: November 18, 2016 (1st); December 9, 2016 (2nd);

December 23, 2016 (3rd); December 28, 2016 (4th).

Accepted: December 30, 2016.

Korean J Anesthesiol 2017 August 70(4): 434-438

https://doi.org/10.4097/kjae.2017.70.4.434

\section{Introduction}

Postoperative pain prevention and treatment continues to be a major challenge in postoperative care and plays an important role in allowing the patient to move and feel better. Although opioid drugs are commonly used in postoperative pain management, they are accompanied by side effects such as nausea, vomiting, drowsiness, itching, and urinary retention, leading to restriction of their use [1]. Other methods such as epidural analgesia are effective, but require extra effort and are associated with serious complications. Nonsteroidal antiinflammatory drugs (NSAIDs) are also used for postoperative analgesia, but may be accompanied by damage to gastrointestinal mucosa,

(c) This is an open-access article distributed under the terms of the Creative Commons Attribution Non-Commercial License (http://creativecommons.org/ licenses/by-nc/4.0/), which permits unrestricted non-commercial use, distribution, and reproduction in any medium, provided the original work is properly cited. 
bleeding, renal toxicity, allergic reactions, and heart failure. Selective cyclooxygenase-2 NSAIDs have pro-thrombotic properties and increase the risk of stroke and myocardial ischemia [2]. A multimodal approach is proposed to relieve postoperative pain and reduce drug side-effects. Considering that surgical stimulation is associated with peripheral and central sensitization, anti-hyperalgesic drugs can treat postoperative pain by preventing central nervous system pain hypersensitivity [3]. Examples of these drugs are gabapentin and pregabalin, which are anti-seizure, anti-hyperalgesic, and anti-anxiety drugs. These drugs bind to the $\alpha 2-\delta-1$ subunit of voltage-dependent calcium channels found in the central nervous system. Pregabalin is structurally similar to gabapentin and induces a greater analgesic effect than gabapentin in the case of neuropathic pain, diabetic peripheral neuropathy [4], and postherpetic neuralgia [5] in animal models. Due to the increased bioavailability ( $90 \%$ vs. 33-66\%), more rapid absorption, and a linear increase in blood concentration with increases in dose, pregabalin is preferred over gabapentin. However, these drugs have side effects such as somnolence, dizziness, nausea, and vomiting.

Gabapentin and recently pregabalin have been used in many studies to control postoperative pain [6]. However, few studies have compared the effects of these drugs on pain control. This study evaluated the effects of pregabalin and gabapentin on postoperative pain in patients undergoing laparoscopic cholecystectomy, and compared them with a placebo group.

\section{Materials and Methods}

In 2014, 108 candidates for laparoscopic cholecystectomy who were between the ages of 20 and 60 with class 1 or 2 American Society of Anesthesiologists and admitted to the Shahid Beheshti Hospital at the Kashan University of Medical Sciences (KAUMS) were enrolled in this randomized, doubleblind clinical trial study. After obtaining university ethics committee approval (NO: 9002) and informed consent, patients were divided into three groups using a random number table: gabapentin $(n=36)$, pregabalin $(n=36)$, or placebo $(n=36)$. Patients received $800 \mathrm{mg}$ of gabapentin or $150 \mathrm{mg}$ of pregabalin orally one hour before surgery in the gabapentin and pregabalin groups, respectively. Patients in the placebo group did not receive either of these medications. In this study, the surgeon, patient, and postoperative pain controller did not know what medication was administered.

After entering the operating room, patients received $10 \mathrm{ml} /$ $\mathrm{kg}$ of Ringer's solution. Heart rate, blood pressure, and blood oxygen saturation was monitored. All patients received $2 \mu \mathrm{g} / \mathrm{kg}$ of fentanyl and $0.05 \mathrm{mg} / \mathrm{kg}$ of midazolam as premedication. After five minutes, anesthesia was induced by thiopental $(6 \mathrm{mg} / \mathrm{kg})$ and $0.5 \mathrm{mg} / \mathrm{kg}$ of atracurium was used to facilitate intubation; patients were then ventilated with $100 \%$ oxygen and intubated with an appropriately sized cuffed tube, after which anesthesia was maintained using $100 \%$ oxygen and isoflurane. Atracurium $(0.2 \mathrm{mg} / \mathrm{kg} / \mathrm{h})$ and fentanyl $(2 \mu \mathrm{g} / \mathrm{kg} / \mathrm{h})$ were used for intraoperative relaxation and analgesia. Capnography was used to measure end-tidal $\mathrm{CO}_{2}$ for all patients and all surgeries were performed by a single surgeon. After surgery, patients were reversed with neostigmine $(70 \mu \mathrm{g} / \mathrm{kg})$ and atropine $(40 \mu \mathrm{g} / \mathrm{kg})$. After extubation and ensuring adequate ventilation, patients were transferred to the recovery care unit for 2 hours and then to the ward. Postoperative pain intensity was measured and recorded based on the visual analogue scale method 2, 6, 12, and 24 hours after surgery. In the cases where the patient felt pain in the recovery room, if the pain score was more than 4 , the patient received 25 $\mathrm{mg}$ of intravenous pethidine. After patients were transferred to the ward, analgesia was administered by pethidine via patientcontrolled analgesia. The amount of opioid consumed, number of nausea events, vomiting, and pain scores at 2, 6, 12, and 24 hours after surgery were recorded. This information, together with the demographic characteristics of the patients and the duration of surgery, were analyzed using SPSS software. Data were analyzed using a repeated measurement test, one-way ANOVA test, chi square test, and post hoc Dunnett's T3 test. P values less than 0.05 were considered statistically significant.

\section{Sample size}

By comparing two mean formulae and comparing $\mu 1, \mu 2$, $\delta 1$, and $\delta 2$ of similar studies for pain intensity [7], $\mu 1, \mu 2, \delta 1$, and $\delta 2$ were (1.99), (3.33), (1.37), and (1.48), respectively, with a $95 \%$ confidence interval and $80 \%$ power for finding the difference between groups. Based on these results, 36 patients in each group were identified.

\section{Results}

In this study, 108 candidates for cholecystectomy were placed in gabapentin, pregabalin, and placebo groups. There were no significant differences between patients in terms of sex, age, duration of surgery, and anesthesia (Table 1).

Comparison of post-operative pain intensity between groups at consecutive times using repeated measurement tests revealed that the pain intensity differed significantly between groups $(\mathrm{P}<0.001)$. The gabapentin and pregabalin groups had lower pain intensity than the placebo group and over time after the surgery the pain intensity in the pregabalin group decreased more than in the gabapentin group (Fig. 1).

Repeated measurement tests on the mean postoperative pethidine consumption revealed a significant interaction between groups $(\mathrm{P}<0.001)$; therefore, a comparison between groups 
Table 1. Frequency Distribution of Patient Gender, Age, Duration of Surgery, and Duration of Anesthesia

\begin{tabular}{lcccc}
\hline & $\begin{array}{c}\text { Gabapentin group } \\
(\mathrm{n}=36)\end{array}$ & $\begin{array}{c}\text { Pregabalin group } \\
(\mathrm{n}=36)\end{array}$ & $\begin{array}{c}\text { Placebo group } \\
(\mathrm{n}=36)\end{array}$ & P value \\
\hline Age & $44.0 \pm 9.5$ & $43.1 \pm 1.1$ & $45.3 \pm 9.3$ & 0.63 \\
Sex (M/F) & $29 / 7$ & $27 / 9$ & $29 / 7$ & 0.57 \\
Duration of surgery (min) & $85.8 \pm 27.1$ & $87.4 \pm 22.1$ & $93.2 \pm 20.7$ & 0.31 \\
Duration of anesthesia (min) & $110.8 \pm 34.5$ & $114.2 \pm 25.4$ & $120.1 \pm 20.4$ & 0.35 \\
\hline
\end{tabular}

Values are expressed as mean \pm SD or number of patients. P value represents a comparison between gabapentin, pregabalin, and placebo groups.

Table 2. Mean Postoperative Opioid (Pethidine, mg) Consumption in Study Groups

\begin{tabular}{lcccc}
\hline & \multicolumn{3}{c}{ Time after surgery } \\
\cline { 2 - 5 } & $2 \mathrm{~h}$ & $6 \mathrm{~h}$ & $12 \mathrm{~h}$ & $24 \mathrm{~h}$ \\
\hline Gabapentin group & $13.2 \pm 1.7^{*}$ & $27.8 \pm 3.5^{*}$ & $37.4 \pm 4.2^{*}$ & $79.9 \pm 1.8^{*}$ \\
Pregabalin group & $11.6 \pm 2.0^{*}$ & $23.7 \pm 3.6^{*}$ & $34.6 \pm 4.2^{*}$ & $78.2 \pm 3.5^{*}$ \\
Placebo group & $15.1 \pm 1.1$ & $30.6 \pm 1.0$ & $42.7 \pm 2.9$ & $89.2 \pm 2.8$ \\
P value & $<0.001$ & $<0.001$ & $<0.001$ & $<0.001$ \\
\hline
\end{tabular}

Values are expressed as mean \pm SD. ${ }^{*} \mathrm{P}<0.001$ compared to the placebo group: one-way ANOVA test.

Table 3. Number of Postoperative Nausea Events in Study Groups

\begin{tabular}{lcccr}
\hline & \multicolumn{3}{c}{ Time after surgery } \\
\cline { 2 - 5 } & $2 \mathrm{~h}$ & $6 \mathrm{~h}$ & $12 \mathrm{~h}$ & $24 \mathrm{~h}$ \\
\hline Gabapentin group & $30(83.3)$ & $28(77.8)^{*}$ & $15(41.7)$ & $7(19.4)$ \\
Pregabalin group & $33(91.7)$ & $28(77.8)^{*}$ & $18(50)$ & $10(27.8)$ \\
Placebo group & $35(97.2)$ & $35(97.2)$ & $21(58.3)$ & $11(30.6)$ \\
P value & 0.12 & 0.03 & 0.36 & 0.53 \\
\hline
\end{tabular}

Values are expressed as number $(\%){ }^{*} \mathrm{P}<0.05$ compared to the placebo group; one-way ANOVA test.

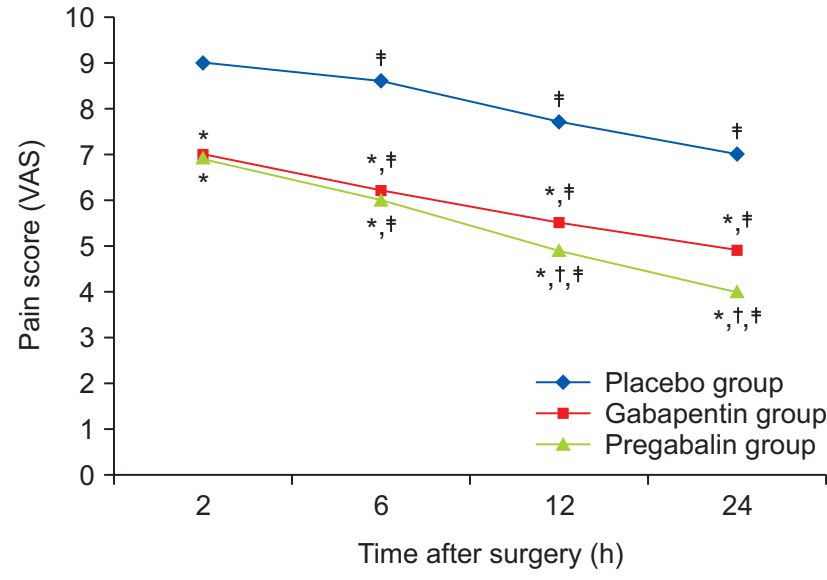

Fig. 1. Comparison of postoperative pain intensity in study groups. ${ }^{*} \mathrm{P}<0.05$ compared with placebo group: one-way ANOVA test, ${ }^{\dagger} \mathrm{P}<0.05$ compared with the Gabapentin group: one-way ANOVA test, ${ }^{\ddagger} \mathrm{P}<0.05$ compared with the group at $2 \mathrm{~h}$ : repeated measurement tests.

was not possible. Pethidine comparison at each individual time (based on a one-way ANOVA test) revealed significant differences between groups. Post-hoc Dunnet's T3 test also revealed that all pairwise comparisons between-groups were significant
Table 4. Distribution of Postoperative Vomiting in Study Groups

\begin{tabular}{llll}
\hline & \multicolumn{2}{c}{ Postoperative vomiting } & \\
\cline { 2 - 3 } & \multicolumn{1}{c}{ Yes } & \multicolumn{1}{c}{ No } & \\
\hline Gabapentin group & $16(55.6)^{*}$ & $20(44.4)^{*}$ & \\
Pregabalin group & $13(36.1)^{*, \dagger}$ & $23(63.9)^{*, \dagger}$ & 0.02 \\
Placebo group & $24(66.7)$ & $12(33.3)$ & \\
\hline
\end{tabular}

Values are expressed as number (\%): chi square test. ${ }^{*} \mathrm{P}<0.05$ compared with the placebo group. ${ }^{\dagger} \mathrm{P}<0.05$ compared with the gabapentin group.

$(\mathrm{P}<0.05)$. Fewer drugs were used in the pregabalin and gabapentin groups, and drug use was lowest in the pregabalin group (Table 2); however, this difference was not significant.

At 6 hours after surgery, both pregabalin and gabapentin groups had significantly less nausea than the control group. No significant differences were observed between the groups in terms of nausea at other time points (Table 3 ).

The pregabalin and gabapentin groups had significantly less vomiting than the placebo group. The pregabalin group had less vomiting than the gabapentin group, and this was statistically significant (Table 4). 


\section{Discussion}

Gabapentin and pregabalin are classified as anti-epileptic drugs, but analgesic and anti-anxiety effects have also been reported [8]. Both drugs have an elimination half-life of 6-8 hours after a single dose. The bioavailability of gabapentin varies with dose and ranges from 35-60\%. At our dose (800 mg), 35-40\% of the drug (300-320 mg) is absorbed, while $90 \%$ of pregabalin is absorbed. Gabapentin reaches its peak effect within 2 hours, while the time to reach the peak effect for pregabalin is $30-120$ minutes [9].

The results of this study revealed that gabapentin $(800 \mathrm{mg}$ ) and pregabalin $(150 \mathrm{mg}$ ) administration before surgery decreased cholecystectomy postoperative pain. Additionally, pain decreased more in the pregabalin group than in the gabapentin group over time.

Several studies have explored the effects of gabapentin on acute postoperative pain, supporting the effectiveness of the drug in reducing pain and lowering opioid use rates after surgery. In a previous report, Forouzanfard et al. [10] compared the effect of 1,200 mg of gabapentin and placebo on pain after abdominal hysterectomy. The results revealed that during the first 24 hours, patients in the gabapentin group experienced less pain and had lower opioid consumption. Other previous reports have supported these results $[11,12]$.

Pregabalin is also effective in controlling acute postoperative pain. Agarwal et al. [13] found that pregabalin significantly decreased pain in patients during movement and at rest after laparoscopic cholecystectomy surgery. These results have been confirmed by Carmichael et al. [14] and Ittichaikulthol et al. [15].

Various studies have observed the effects of gabapentin or pregabalin on reducing pain and opioid consumption in patients undergoing surgery, but limited studies have compared the effects of gabapentin and pregabalin on postoperative pain. In a previous report, Ozgencil et al. [6] compared the effects of pregabalin, gabapentin, and placebo on post-operative pain. They found that postoperative pain was lower in the gabapentin and pregabalin groups than the placebo group at 6 hours after surgery, but observed no significant differences between the three groups at 12 and 24 hours. Morphine consumption was significantly lower in the gabapentin and pregabalin groups compared to the placebo group. In our study, the pain difference continued until 24 hours after surgery. This may be due to increased consumption of morphine in Ozgencil's study in the placebo group after the 6-hour time point compared to the other two groups. Ghai et al. [9] gave patients $300 \mathrm{mg}$ of pregabalin, $900 \mathrm{mg}$ of gabapentin, or placebo 2 hours prior to hysterectomy. Muscular diclofenac and tramadol were used for postoperative analgesia. They found that the length of analgesic demand and analgesic consumption were both lowest in the pregabalin group, which supports our results.

Several animal and human studies have reported that mechanical damage to nerves increases neural discharge to the central nervous system, leading to the 'central sensitization' process [16]; gabapentin and pregabalin may interfere with pain processing, leading to sensitization of the central nervous system and decreasing the experience of pain and neuropathic pain [17]. An imaging study using functional MRI revealed that gabapentin decreased activity of the brainstem during central sensitization. Additionally, gabapentin suppressed the stimuli, leading to deactivation (from other regions of the brain) during central sensitization. This indicates that pain modulation by gabapentin occurs in the presence of central sensitization [18]. Gabapentin and pregabalin may exert their effects through a2- $\delta-1$ protein subunits of voltage-dependent calcium channels, which are dense in the posterior horn of the spinal cord rather than the anti-inflammatory compounds $[19,20]$. Another mechanism that leads to pain relief through the drugs' effects on a2- $\delta$ is their effect on the descending regulatory pathway [21]. Finally, local changes in the function of spinal norepinephrine induced by these compounds may regulate pre- or post-synaptic afferent input and potentially lead to clinical adjustment of pain [22].

Nausea and vomiting are postoperative concerns, and about $53-72 \%$ of patients require postoperative anti-nausea and antivomiting treatment after laparoscopic cholecystectomy [23]. In this study, nausea was significantly lower in the gabapentin and pregabalin groups than the placebo group at 2-6 hours after surgery; no significant differences were observed at other times. The number of cases of vomiting was significantly lower in the gabapentin and pregabalin groups than in the placebo group. In a previous study involving 250 patients undergoing laparoscopic cholecystectomy, postoperative nausea and vomiting in the group that had received $600 \mathrm{mg}$ of gabapentin were significantly lower than in the placebo group [24]. Two systematically conducted surveys have also shown that nausea and vomiting in cases of gabapentin and pregabalin administration was lower than in the placebo group $[2,25]$. The mechanism of gabapentin on postoperative vomiting remains unknown, but there are two possible causes: 1) an indirect effect that reduces postoperative nausea and vomiting by decreasing opioid consumption; and 2) a direct mechanism that decreases postoperative nausea and vomiting by decreasing tachykinin neurotransmitter activity [26].

The results of this study revealed that gabapentin and pregabalin were effective in controlling laparoscopic cholecystectomy postoperative pain, nausea, and vomiting, and compared with the placebo group, decreased opioid consumption. Moreover, the findings revealed that pregabalin is superior to gabapentin for reduced postoperative pain and opioid consumption. 


\section{Acknowledgments}

This study was conducted as a surgeon's assistant's thesis supported by the Deputy of Research, Kashan University of Medical
Sciences (grant number: 90002). We would also like to thank the staff at the Shahid Beheshti Hospital at the Kashan University of Medical Sciences (KAUMS) for their help and cooperation.

\section{References}

1. Dolin SJ, Cashman JN. Tolerability of acute postoperative pain management: nausea, vomiting, sedation, pruritus, and urinary retention. Evidence from published data. Br J Anaesth 2005; 95: 584-91.

2. Tiippana EM, Hamunen K, Kontinen VK, Kalso E. Do surgical patients benefit from perioperative gabapentin/pregabalin? A systematic review of efficacy and safety. Anesth Analg 2007; 104: 1545-56.

3. Woolf CJ, Chong MS. Preemptive analgesia--treating postoperative pain by preventing the establishment of central sensitization. Anesth Analg 1993; 77: 362-79.

4. Frampton JE, Scott LJ. Pregabalin: in the treatment of painful diabetic peripheral neuropathy. Drugs 2004; 64: 2813-20.

5. Frampton JE1, Foster RH. Pregabalin: in the treatment of postherpetic neuralgia. Drugs 2005; 65: 111-8.

6. Ozgencil E, Yalcin S, Tuna H, Yorukoglu D, Kecik Y. Perioperative administration of gabapentin 1,200 mg day-1 and pregabalin $300 \mathrm{mg}$ day-1 for pain following lumbar laminectomy and discectomy: a randomised, double-blinded, placebo-controlled study. Singapore Med J 2011 ; 52: 883-9.

7. Pandey CK, Priye S, Singh S, Singh U, Singh RB, Singh PK. Preemptive use of gabapentin significantly decreases postoperative pain and rescue analgesic requirements in laparoscopic cholecystectomy. Can J Anaesth 2004; 51: 358-63.

8. Imani F, Rahimzadeh P. Gabapentinoids: gabapentin and pregabalin for postoperative pain management. Anesth Pain Med 2012; 2: 52-3.

9. Ghai A, Gupta M, Hooda S, Singla D, Wadhera R. A randomized controlled trial to compare pregabalin with gabapentin for postoperative pain in abdominal hysterectomy. Saudi J Anaesth 2011; 5: 252-7.

10. Frouzanfard F, Fazel MR, Abolhasani A, Fakharian E, Mousavi G, Moravveji A. Effects of gabapentin on pain and opioid consumption after abdominal hysterectomy. Pain Res Manag 2013; 18: 94-6.

11. Yeganeh Mogadam A, Fazel MR, Parviz S. Comparison of analgesic effect between gabapentin and diclofenac on post-operative pain in patients undergoing tonsillectomy. Arch Trauma Res 2012; 1: 108-11.

12. Dirks J, Fredensborg BB, Christensen D, Fomsgaard JS, Flyger H, Dahl JB. A randomized study of the effects of single-dose gabapentin versus placebo on postoperative pain and morphine consumption after mastectomy. Anesthesiology 2002; 97: 560-4.

13. Agarwal A, Gautam S, Gupta D, Agarwal S, Singh PK, Singh U. Evaluation of a single preoperative dose of pregabalin for attenuation of postoperative pain after laparoscopic cholecystectomy. Br J Anaesth 2008; 101: 700-4.

14. Carmichael NM, Katz J, Clarke H, Kennedy D, Kreder HJ, Gollish J, et al. An intensive perioperative regimen of pregabalin and celecoxib reduces pain and improves physical function scores six weeks after total hip arthroplasty: a prospective randomized controlled trial. Pain Res Manag 2013; 18: 127-32.

15. Ittichaikulthol W, Virankabutra T, Kunopart M, Khamhom W, Putarawuthichai P, Rungphet S. Effects of pregabalin on post operative morphine consumption and pain after abdominal hysterectomy with/without salphingo-oophorectomy: a randomized, double-blind trial. J Med Assoc Thai 2009; 92: 1318-23.

16. Ohnami S, Tanabe M, Shinohara S, Takasu K, Kato A, Ono H. Role of voltage-dependent calcium channel subtypes in spinal long-term potentiation of C-fiber-evoked field potentials. Pain 2011; 152: 623-31.

17. Woolf CJ. Central sensitization: implications for the diagnosis and treatment of pain. Pain 2011; 152(3 Suppl): S2-15.

18. Tuchman M, Barrett JA, Donevan S, Hedberg TG, Taylor CP. Central sensitization and $\mathrm{Ca}(\mathrm{V}) \alpha_{2} \delta$ ligands in chronic pain syndromes: pathologic processes and pharmacologic effect. J Pain 2010; 11: 1241-9.

19. Stahl SM, Porreca F, Taylor CP, Cheung R, Thorpe AJ, Clair A. The diverse therapeutic actions of pregabalin: is a single mechanism responsible for several pharmacological activities? Trends Pharmacol Sci 2013; 34: 332-9.

20. Field MJ, Cox PJ, Stott E, Melrose H, Offord J, Su TZ, et al. Identification of the alpha2-delta-1 subunit of voltage-dependent calcium channels as a molecular target for pain mediating the analgesic actions of pregabalin. Proc Natl Acad Sci U S A 2006; 103: $17537-42$.

21. Ossipov MH, Dussor GO, Porreca F. Central modulation of pain. J Clin Invest 2010; 120: 3779-87.

22. Hayashida K, Eisenach JC. A tropomyosine receptor kinase inhibitor blocks spinal neuroplasticity essential for the anti-hypersensitivity effects of gabapentin and clonidine in rats with peripheral nerve injury. J Pain 2011; 12: 94-100.

23. Gupta P, Khanna J, Mitramustafi AK, Bhartia VK. Role of pre-operative dexamethasone as prophylaxis for postoperative nausea and vomiting in laparoscopic surgery. J Minim Access Surg 2006; 2: 12-5.

24. Pandey CK, Priye S, Ambesh SP, Singh S, Singh U, Singh PK. Prophylactic gabapentin for prevention of postoperative nausea and vomiting in patients undergoing laparoscopic cholecystectomy: a randomized, double-blind, placebo-controlled study. J Postgrad Med 2006; 52: 97-100.

25. Ho KY, Gan TJ, Habib AS. Gabapentin and postoperative pain--a systematic review of randomized controlled trials. Pain 2006; $126: 91-101$.

26. Kong VK, Irwin MG. Gabapentin: a multimodal perioperative drug? Br J Anaesth 2007; 99: 775-86. 